\title{
Acinetobacter baumannii antibiotics resistance in Iran
}

\begin{abstract}
One of the causes of nosocomial infection is Acinetobacter baumannii that is a gramnegative coccobacillus bacteria, especially in intensive care units (ICUs), which can produce various types of infections. The recent emergence of Multiple drug resistance (MDR) A. baumannii has become serious trouble and these MDR strains are quickly distributed among hospitalized patients. We performed a literature review published on antibiotic-resistant clinical A. baumannii isolates reported in Iran from 2012 to 2017. There was an increase in resistance to gentamicin, imipenem, meropenem, piperacillin, ampicillin/sulbactam, ticarcillin, tobramycin, aztreonam .This study indicates that drugresistance against several antibiotics is occurring for A. baumannii in Iran. Traveling to high prevalence countries and overuse of antimicrobial agents could be the factors contributing to the high prevalence of resistance. To stop occurring this phenomenon, suitable solutions and accurate preventions must be taken.
\end{abstract}

Volume 7 Issue 6 - 2019

\author{
Atefeh Hamzeh,' Parastoo Rezaee, ${ }^{2}$ Mahsa \\ Mohammadi ${ }^{3}$ \\ 'Department of Biotechnology, Alzahra University, Iran \\ ${ }^{2}$ Department of Microbiology, Alzahra University, Iran \\ ${ }^{3}$ Quality Control Management, Iran
}

Correspondence: Parastoo Rezaee, Master of Microbial Biotechnology, Department of Biotechnology, Faculty of Biology, Alzahra University,Tehran, Iran, Email parastreza@gmail.com Received: November 08, 2019 | Published: December 20,
2019

Keywords: acinetobacter baumannii, Iran, antibiotic resistance

\section{Objectives}

One of the causes of nosocomial infection is A. baumannii that is a gram-negative coccobacillus bacteria, especially in intensive care units (ICUs). It causes ventilator-associated pneumonia, bacteremia, surgical-site infections, secondary meningitis, and urinary tract infections..$^{1-3}$ Several factors may contribute to the virulence potential of $A$. baumannii such as OmpA, Pili, biofilm-associated protein (BAP) and phospholipase D and C.4-6 The recent emergence of MDR A. baumannii has become serious trouble and these MDR strains are quickly distributed among hospitalized patients. Three main mechanisms of antibiotics resistance involve disabling antibiotics with enzymes, decline entrance antibiotics into the target site of bacteria and alteration of the target or cellular functions. ${ }^{7,8}$ The World Health Organization (WHO) has published a list of priority pathogens that are resistant to antibiotics for research and development about new antibiotics, including $A$. baumannii, this shows the importance of antibiotic resistance of this bacterium. ${ }^{9}$ Therefore, to investigate the development of antibiotic resistance of this bacterium in Iran, we reviewed articles published in this regard in the years 2012 to 2017.

\section{Methods}

To gather the necessary information from published articles from national and international journals, research in various databases was reviewed, including PubMed, Google Scholar, Scopus, SID from 2012 to 2017. Keywords for searching articles, inclusive "antibiotic, drag or antimicrobial resistance" with different combinations of other key text words such as A. baumannii and Iran. After analyzing the related articles, were categorized according to year and antibiotic for a better conclusion. Antibiotics investigated included Aminoglycoside, Fluoroquinolones, Penicillin, Carbapenem, Cephalosporin, Miscellaneous and Macrolide.

\section{Results}

We reviewed Thirty-six related articles that included $3011 \mathrm{~A}$. baumannii clinical isolates and 25 antibiotics from 2012 to $2017 .^{10-45}$ Antibiotic resistance effect methods in investigated articles include disc diffusion assay (Kirby-Bauer) and molecular method (PCR). The data of antibiotic resistance of 3011 A. baumannii isolates are presented in Table 1. This information demonstrates that the resistance to amikacin, ciprofloxacin, levofloxacin, piperacillin, ticarcillin, ceftazidime, ceftriaxone, cefotaxime, cefepime, trimethoprim/ sulfamethoxazole were high, the resistance to gentamicin, tetracycline, tobramycin, ampicillin/sulbactam, aztreonam, meropenem, imipenem were increased in these years and resistance to polymyxin B \& colistin was less or decreased.

Table I Percentage of antibiotic resistance in clinical Acinetobacter baumannii isolates in Iran from 2012 to 2017

\begin{tabular}{|c|c|c|c|}
\hline \multirow{4}{*}{ Antibiotic agents } & \multicolumn{3}{|c|}{ Mean resistance rate $(\%)$ in studies reviewed } \\
\hline & $2016-2017$ & $2014-2015$ & $2012-2013$ \\
\hline & $\mathrm{n}=467$ & $\mathrm{n}=992$ & $n=1552$ \\
\hline & (\%) & (\%) & (\%) \\
\hline Amikacin & 76.5 & 76.88 & 83.93 \\
\hline Gentamicin & 87.62 & 77.16 & 75.43 \\
\hline Tetracycline & 89.5 & 78.3 & 73 \\
\hline
\end{tabular}




\begin{tabular}{|c|c|c|c|}
\hline \multirow{4}{*}{ Antibiotic agents } & \multicolumn{3}{|c|}{ Mean resistance rate $(\%)$ in studies reviewed } \\
\hline & $2016-2017$ & $20|4-20| 5$ & $2012-2013$ \\
\hline & $n=467$ & $n=992$ & $n=\mid 552$ \\
\hline & (\%) & (\%) & (\%) \\
\hline Tobramycin & 83 & 64.44 & 48.6 \\
\hline Tigecycline & 15 & - & 48 \\
\hline Ciprofloxacin & 99.83 & 86.56 & 92.88 \\
\hline Levofloxacin & 99.75 & 61.8 & 96.13 \\
\hline Piperacillin & 100 & 97.73 & 95.17 \\
\hline Pip/tazo & 86.6 & 96.48 & 90.89 \\
\hline Ampi/sulba & 95.5 & 80.47 & 73.45 \\
\hline Ticarcillin & 100 & - & 97.63 \\
\hline Aztreonam & 100 & - & 76.63 \\
\hline Imipenem & 86.62 & 76.88 & 76.4 \\
\hline Meropenem & 99.75 & 77.8 & 78.62 \\
\hline Ceftazidime & 92.5 & 86.69 & 94.97 \\
\hline Ceftriaxone & 76 & 85.72 & 97.23 \\
\hline Cefotaxime & 100 & 99.8 & 95.76 \\
\hline Cefepime & - & 94.04 & 95.83 \\
\hline Polymyxin B & 0 & 6.39 & 3.54 \\
\hline Colistin & 6 & 8.31 & 22.84 \\
\hline Trim/sulfa & - & 81.34 & 84.5 \\
\hline
\end{tabular}

\section{Discussion and conclusion}

A. baumannii colonization is important in patients with intubation who have multiple intravenous lines or surveillance devices, surgical drainage, or in vivo urinary catheters. ${ }^{44}$ Controlling of $A$. baumannii infection is very difficult because of its resistance to different antimicrobial agents, despite resistance to imipenem, it is still the drug to selection. ${ }^{45}$ The reports also showed that carbapenem-resistant isolates are increasing worldwide. ${ }^{46-48}$ Currently, lipopeptides antibiotics such as polymyxin B and colistin are used to treat MDR A. baumannii. ${ }^{22,49}$ According to studies in Iran, the average is $71 \% .{ }^{50}$ This prevalence is $67 \%$ in Iraq, $83 \%$ in the United Arab Emirates, $85 \%$ in Kuwait, $100 \%$ Pakistan, $74.2 \%$ in India, $98.9 \%$ in Turkey ${ }^{33}$ so one of the reasons for the high prevalence in Iran could be traveling to these countries. The overuse of antimicrobial agents is also effective. This study indicates that the antibiotic resistance of $A$. baumannii is high in Iran. Therefore, it is necessary to implement appropriate solutions and preventive programs.

\section{Funding details}

None.

\section{Acknowledgements}

None.

\section{Conflicts of interest}

The author declares no conflict of interest.

\section{References}

1. Peleg AY, Seifert H, Paterson DL. Acinetobacter baumannii: emergence of a successful pathogen. Clin Microbiol Rev. 2008;21:538-582.

2. Jung J, Park W. Acinetobacter species as model microorganisms in environmental microbiology: current state and perspectives. Appl Microbiol Biotechnol. 2015;99:2533-2548.

3. Doughari HJ, Ndakidemi PA, Human IS, et al. The ecology, biology and pathogenesis of Acinetobacter spp: an overview. Microbes Environ. 2011;26:101-112.

4. Howard A, O'Donoghue M, Feeney A, et al. Acinetobacter baumannii: an emerging opportunistic pathogen. Virulence. 2012;3(3):243-50.

5. Gaddy JA, Actis LA. Regulation of Acinetobacter baumannii biofilm formation. Future Microbiol. 2009;4:273-278.

6. Jacobs AC, Hood I, Boyd KL, et al. Inactivation of phospholipase D diminishes Acinetobacter baumannii pathogenesis. Infect Immun. 2010;78:1952-1962.

7. Rice LB. Challenges in identifying new antimicrobial agents effective for treating infections with Acinetobacter baumannii and Pseudomonas aeruginosa. Clin Infect Dis. 2006;43:100-105. 
8. Lee C, Lee J, Park M, et al. Biology of Acinetobacter baumannii: Pathogenesis, Antibiotic Resistance Mechanisms, and Prospective Treatment Options. Frontiers in Cellular and Infection Microbiology. 2017;10(55):1-35.

9. Organization WHO. WHO Publishes List of Bacteria for which New Antibiotics are Urgently Needed. Geneva,: WHO; 2017.

10. Ahmadi K, Mardaneh J, Saadat S. Determination antimicrobial resistance profile of Acinetobacter strains isolated from hospitalized patients in Different Part of Taleghani Hospital (Ahvaz, Iran). ISMJ. 2014;15;17:620-628.

11. Jafari S, Najafipour S, Kargar M, et al. Phenotypical evaluation of multidrug resistant Acinetobacter baumannii. Journal of Fasa University of Medical Sciences. 2013;15:254-258.

12. Dehbalaei MA, Najar-Peerayeh S, Taherikalani M, et al. Clinical isolates of Acinetobacter baumannii from Tehran hospitals: Pulsedfield gel electrophoresis characterization, clonal lineages, antibiotic susceptibility, and biofilm-forming ability. Jundishapur Journal of Microbiology. 2017; 10:13790.

13. Najar Peerayeh S, Karmostaji A, Salmanian AH. Distribution of OXAtype class D $\beta$-lactamase genes among nosocomial multi drug resistant Acinetobacter baumannii isolated in Tehran hospitals. Jundishapur Journal of Microbiology. 2013;6:1-5.

14. Vafaei S, Mirnejad R, Amirmozafari N. Determining the Patterns of Antimicrobial Susceptibility and the Distribution of blaCTX-M Genes in Strains of Acinetobacter Baumannii Isolated from Clinical Samples. Journal of Isfahan Medical School. 2013;8:155-160.

15. Aliakbarzade K, Farajnia S, Nik AK, et al. Prevalence of aminoglycoside resistance genes in Acinetobacter baumannii isolates. Jundishapur journal of microbiology. 2014;7(10):11924.

16. Yousefian R, Karbasizade V, Moghim Sh. Identification and Frequency of Colistin Resistant Acintobacterbaumanii from Clinical Isolates By PCR. J Isfahan Med Sch. 2014;32(301).

17. Soltani R, Ehsanpoor M, Khorvash F, et al. Antimicrobial susceptibility pattern of extended-spectrum $\beta$-lactamase-producing bacteria causing nosocomial urinary tract infections in an Iranian referral teaching hospital. Journal of research in pharmacy practice. 2014 ;3(1):6.

18. Sarhaddi N, Dolatabadi S, Amel Jamehdar S. Drug resistance pattern of carbapenem-resistant Acinetobacter baumannii isolated from a referral burn center in northeast of Iran. Medical journal of mashhad university of medical sciences. 2016;59(1):1-8.

19. Babapour E, Haddadi A, Mirnejad R, et al. Biofilm formation in clinical isolates of nosocomial Acinetobacter baumannii and its relationship with multidrug resistance. Asian Pacific Journal of Tropical Biomedicine. 2016;6(6):528-533.

20. Safari M, Saidijam M, Bahador A, et al. High prevalence of multidrug resistance and metallo-beta-lactamase $(\mathrm{M} \beta \mathrm{L})$ producing Acinetobacter baumannii isolated from patients in ICU wards, Hamadan, Iran. Journal of research in health sciences. 2013;13(2):162-167.

21. Fallah F, Noori M, Hashemi A, et al. Prevalence of blaNDM, blaPER, blaVEB, blaIMP, and blaVIM genes among Acinetobacter baumannii isolated from two hospitals of Tehran, Iran. Scientifica. 2014.

22. Vakili B, Fazeli H, Shoaei P, et al. Detection of colistin sensitivity in clinical isolates of Acinetobacter baumannii in Iran. Iran J Res Med Sci. 2014; 19:67-70

23. Kamalbeik S, Talaie H, Mahdavinejad A, et al. Multidrug-resistan Acinetobacter baumannii infection in intensive care unit patients in a hospital with building construction: is there an association? Korean $J$ Anesthesiol. 2014;66(4):295-299.
24. Josheghani SB, Moniri R, Firoozeh F, et al. Emergence of bla OXACarrying Carbapenem Resistance in Multidrug-Resistant Acinetobacter baumannii in the Intensive Care Unit. Iranian Red Crescent Medical Journal. 2017;19(5);e27327.

25. Sarikhani Z, Nazari R, Rostami MN. First report of OXA-143-lactamase producing Acinetobacter baumannii in Qom, Iran. Iranian journal of basic medical sciences. 2017;20(11):1282.

26. Khaledi A, Bahador A, Mansoori M, et al. Determination of antimicrobial resistance pattern of Acinetobacter baumanii isolated from patients in intensive care unit (ICU). Medical Journal Of Mashhad University Of Medical Sciences. 2015;58(7);376-380.

27. Goudarzi H, Doraghi M, Dabiri H, et al. Functional analysis of multidrug efflux pumps genes of Acinetobacter baumannii strains. Research in Medicine. 2013;37(2):107-112.

28. Nazari A, Hosseini Doust R, et al. Prevalance per and veb beta lactamase genes among Acintobacter baumannii Isolates from patients in Tehran by PCR. Iranian J of Med Mic. 2014;8(4):28-35.

29. Sayehmiri F, Alikhani M, Sayehmiri K, et al. The prevalence of antibiotic of Acintobacter baumannii Iran and the world: a systematic Review and meta-analysis. Shiraz E-Med J. 2017;18(2):1-8.

30. Kooti S, Mohammad M, Sarvari J. Antibiotic resistance profile and distribution of oxacillinase genes among clinical isolates of Acintobacter baumannii in shiraz teaching hospital, 2012-2013. Jundishapur $J$ Microbial. 2015; 8(8):1-6.

31. Esmaeilzadeh Ashini E, Amini K. Isolation of tetD, tetC, tetB, tetA from Acintobacter baumannii samples isolates from hospital samples by multiplex PCR method. Medical J of Mashahd. 2017;60(6):734-742.

32. Zahedi, Bialvaei A, Kouhsari E, et al. Epidemiology of multidrugresistant Acinetobacterbaumannii strains in Iran: a systematic review and meta-analysis. Journal of Chemotherapy. 2017;1(31):1-11.

33. Pourhajibagher M, Hashemi F, Pourakbari B, et al. Antimicrobial Resistance of Acinetobacter baumannii to Imipenem in Iran: A Systematic Review and Meta-Analysis. The Open Microbiology Journal. 2016;10:32-42.

34. Nourbakhsh F, Nourbakhsh V, Jafakesh MT. Prevalence of class I, II and III integrons in the antibiotic-resistant isolates of $A$. baumannii detected from patients hospitalized in medical centers of Shahrekord. Feyz Journal of Kashan University of Medical Sciences. 2016;20.

35. Khoshnood S, Eslami G, Hashemi A, et al. Distribution of aminoglycoside resistance genes among Acinetobacter baumannii strains isolated from burn patients in Tehran, Iran. Archives of Pediatric Infectious Diseases. 2017;5(3):e57263.

36. Tavakol M, Momtaz H. Detection of the most prevalent antibiotic resistance genes in Acinetobacter baumannii strains isolated from hospital infections and determination of their antibiotic resistance pattern. Biology of microorganism. 2015;4:71-82.

37. Madadi-Goli N, Moniri R, Bagheri-Josheghani S. Antibiotic resistance pattern and distribution of Vietnamese extendedspectrum- $\beta$ lactamase (VEB-1) gene in Acinetobacter baumannii isolatedfrom hospitalized patients in KashanShahidBeheshti hospital during2013-2014. Journal of Kashan University of Medical Sciences. 2017;21:383-389.

38. Ardeshiri N, Nasrollahi M , Goudarzi H, et al. The prevalence of Integron 1, 2 and 3 classes in Acinetobacter baumanii clinical isolates from Sari Hospitals, Iran. Iran. Research in Medicine. 2017;41:217-225.

39. Moezi H, Javadpour S, Golestani F. Identification of Different Species of Acinetobacter Strains, and Determination ofTheir Antibiotic Resistance Pattern and MIC of Carbapenems by E-Test. Hormozgan Medical Journal. 2016;20:45-51. 
40. Rezai MS, Rafiei A, Ahangarkani F, et al. Emergence of Extensively Drug Resistant Acinetobacter baumannii-Encoding Integrons and ExtendedSpectrum Beta-Lactamase Genes Isolated from Ventilator-Associated Pneumonia Patients. Jundishapur J Microbiol. 2017;10(7):e14377.

41. Darvishi M. Virulence Factors Profile and Antimicrobial Resistance of Acinetobacter baumannii Strains Isolated from Various Infections Recovered from Immunosuppressive Patients. Biomedical \& Pharmacology Journal. 2016;9:1057-1062.

42. Soltani B, Heidari H, Ebrahim-Saraie HS, et al. Molecular characteristics of multiple and extensive drug-resistant Acinetobacter baumannii isolates obtained from hospitalized patients in Southwestern Iran. Infez Med. 2018;26(1):67-76.

43. Deylam Salehi M, Ferdosi-Shahandashti E, Yahyapour Y, et al. IntegronMediated Antibiotic Resistance in Acinetobacter baumannii Isolated from Intensive Care Unit Patients, Babol, North of Iran. BioMed Research International. 2017;10:1-8.

44. Cisneros JM, Rodríguez-Baño. Nosocomial bacteremia due to Acinetobacter baumannii: epidemiology, clinical features and treatment. JClin Microbiol Infect. 2002;8(11):687-693.

45. Perez F, Hujer AM, Hujer KM, et al. Global challenge of multidrugresistant Acinetobacter baumannii. Antimicrob Agents Chemother. 2007;51(10):3471-3484.
46. Beigverdi R, Sattari-Maraji A, Emaneini M, et al. Status of carbapenemresistant Acinetobacter baumannii harboring carbapenemase: First systematic review and meta-analysis from Iran. Infect Genet Evol. 2019;73:433-443.

47. Yazdansetad S, Najari E, Ghaemi EA, et al. Carbapenem-resistant Acinetobacter baumannii isolates carrying blaOXA genes with upstream ISAba1: First report of a novel OXA subclass from Iran. $J$ Glob Antimicrob Resist. 2019;18:95-99.

48. Ozcan N, Dal T, Şukran Can, et al. OXA Carbapenemase Genes in Multidrug-Resistant A. baumannii Strains in Southeast of Turkey. SMU Medical Journal. 2015;2(2):21-32.

49. Mirnejad R, Heidary M, Bahramian A, et al. Evaluation of Polymyxin B Susceptibility Profile and Detection of Drug Resistance Genes among Acinetobacter Baumannii Clinical Isolates in Tehran, Iran during 20152016. Mediterr J Hematol Infect Dis. 2018;10(1):e2018044.

50. Moradi J, Hashemi FB, Bahador A. Antibiotic Resistance of Acinetobacter baumannii in Iran: A Systemic Review of the Published Literature. Osong Public Health Res Perspect. 2015;6(2):79-86. 